\title{
Deterministic and stochastic processes in natural and modified floodplain habitats: consequences for fish community temporal dynamics
}

\author{
Bin $\mathrm{Li}^{1}$, Yuyu Wang ${ }^{1}$, Wenzhuo Tan ${ }^{1}$, Neil Saintilan ${ }^{2}$, Guangchun Lei ${ }^{1}$, and Li Wen ${ }^{1}$ \\ ${ }^{1}$ Beijing Forestry University \\ ${ }^{2}$ Macquarie University Faculty of Science
}

July 16, 2020

\begin{abstract}
Habitat degradation is expected to alter community structure and consequently, ecosystem functions including the maintenance of biodiversity. Understanding the underlying abiotic and biotic assembly mechanisms controlling temporal and spatial community structure and patterns is a central issue in biodiversity conservation. In this study, using monthly time series of benthic fish data collected over a three-year period, we compared the temporal community dynamics in natural and modified habitats in one of the largest river-lake floodplain ecosystems in China, the Dongting Lake. We found a prevailing strong positive species covariance, i.e. species abundance changes in the same way, in all communities that was significantly negatively impacted by water nutrients levels. The positive species covariance, which was consistent for both wet and dry years and among habitat types, had significantly negative effects on community stability, which was measured by the average of aggregated abundance divided by temporal standard deviation. In contrast to species covariance, community stability was significantly higher in modified habitats than in natural habitats. Furthermore, our results demonstrated that the ecological stochasticity (i.e. community assembly processes generating diversity patterns that are indistinguishable from random chance) was significantly higher at natural sites than at the modified sites, suggesting that deterministic processes might control the community composition (richness and abundance) at the modified habitat through reducing species synchrony and positive species covariance observed in the natural habitats. When combined, our results suggest that human habitat modification creates environmental conditions for the development of stable benthic fish community in the highly dynamic floodplains, leading to niche-based community and decrease of temporal $\beta$-diversity.
\end{abstract}

\section{Introduction}

Globally, freshwater ecosystems have undergone massive changes and the increasing loss of freshwater biodiversity is widespread (Palmer \& Ruhí 2019; Reid et al. 2019). Floodplain ecosystems of large river are some of the most heavily exploited and valued ecosystems and also among the most modified and degraded (Bayley 1995; Vörösmarty et al. 2010; Castello \& Macedo 2016). Human modifications such as river regulation and land reclamation have greatly changed the inundation regimes such as extent, duration, timing and frequency (Poff et al. 1997, 2006; Olden \& Naiman 2010; Lu et al. 2018); and have diminished the benefits of hydrological connectivity for vegetation (Guan et al. 2016), fishes (Fontenot et al. 2001; Li et al. 2020), waterbirds (Xia et al. 2017) and many other aquatic organisms (Kupferberg et al. 2012). Thus, floodplain system restoration programs, such as environmental water allocation (Stewart \& Harper 2002), reconnecting floodplain with its rivers (Pander et al. 2015) and returning croplands to wetland (Xu et al. 2018), remain a focus of regional biodiversity conservation. 
Understanding the underlying abiotic and biotic assembly mechanisms controlling temporal and spatial community structure and patterns is not only a central issue in ecology (Cottenie 2005; Leibold et al. 2004; Magurran et al., 2018 \& 2019; McGill 2003; Ning et al. 2019), but also has practical implications for biodiversity conservation (Economo 2011; McGill et al. 2015; Yurkonis et al. 2005). Many mechanisms have been hypothesized as explaining spatial and temporal variations in community structure, i.e. the number of taxa (richness) and their relative abundance (composition). The current advances in metacommunity theory generally suggest that the two broad types of processes, i.e. deterministic and stochastic, work simultaneously in shaping ecological communities (Chase \& Myers 2011; Gaston \& Chown 2005; Gravel et al. 2006; Daniel et al. 2019). The deterministic assembly generally refers to ecological niche-based mechanisms, including environmental filtering (e.g., climate, $\mathrm{pH}$, nutrients, and salinity) and biological interactions (e.g., competition and facilitation) (Levi et al. 2019; Silvertown 2004). Considering that all species are ecologically equivalent in the probabilistic sense (i.e. individuals have equal chances of reproduction or death, Hubbell 2001; Rosindell et al. 2012), the stochastic processes, however, concerns ecological processes that generate community patterns indistinguishable from neutral simulations through random birth- death events, probabilistic dispersal, and ecological drift (random walk, either to extinction or monodominance) (Hubbell 2001; Gravel et al. 2006; Ning et al. 2019; Zhou et al. 2014). A range of methods have been used to quantify the relative importance of deterministic and stochastic processes in community assembly (Anderson et al. 2011; Baselga 2010), of which the null model approach is the most widely used (Mori et al. 2015; Mori et al. 2013). Through randomizing the empirical community matrix (Gotelli 2000), null models can detect if and by how much the observed variation in community structure (i.e. $\beta$-diversity) deviates from the null expectation given a regional species pool (i.e. gamma diversity) (Chase et al. 2011). Large deviations are suggestive of deterministic processes driving community assembly while close to zero deviations are interpreted as neutral community (Gotelli 2000; Tucker et al. 2016).

Research on the temporal dimensions of community ecology is becoming increasingly urgent in the face of accelerating loss of biodiversity in the Anthropocene (Dirzo et al. 2014; Garcia-Moreno et al. 2014; McGill et al. 2015). The study of temporal dynamics can help to enhance our predictions on the responses of communities to natural environmental fluctuations and anthropogenic disruptions (Collins et al. 2000; Kéfi et al. 2019; Rosset et al. 2017) by revealing how species respond to community-level constraints (Musters et al. 2019) and how species interactions change over time (Gonzalez \& Loreau 2009; Hallett et al. 2014). This knowledge has strong conservation implications. For example, identifying keystone communities that disproportionally contribute to the maintenance of regional diversity is critical to conservation planning (Ruhí et al. 2017). Many statistical metrics and analytical tools, such as community stability (Tilman 1999), species turnover and community change rate (Collins et al. 2000; Collins et al. 2008), synchrony (Hallett et al. 2016) and temporal beta-diversity index (Legendre 2019) have been developed to quantify and compare the temporal dynamics.

Over the last two decades, studies on spatial variation in community structure (i.e. spatial beta-diversity) have played a central role in understanding how communities are organized along environmental gradients, and how stochastic and deterministic processes influence the functioning of ecosystems (Jia et al. 2020; Heino et al. 2015; Mori et al. 2018). In comparison, far fewer studies have investigated the temporal dynamics of communities (see Korhonen et al. 2010 and Jones et al. 2017 for examples), limiting our understanding on how communities respond to the widespread and rapid changes in the Anthropocene (Dirzo et al. 2014; Ruhí et al. 2017), which may lead to contradictory predictions (see Gonzalez et al. 2016 and Vellend et al. 2017). This limitation is particularly significant for the highly variable ecosystems, such as floodplains, where communities exhibit high intra- and inter- annual variability in community assembly processes (Heino et al. 2015).

Despite decades of study, quantifying the relative importance of deterministic versus stochastic processes in natural community assembly remains a key challenge to ecologists (Dini-Andreote et al. 2015; Tucker et al. 2016), especially in dynamic species-rich landscapes (Ruhí et al. 2017). In this study, we collected monthly samples of benthic fish communities in 12 sites, of which six are located at highly modifiedPopulus nigra plantations and six are from natural Carexsedges and open waters, over three consecutive years in 
a large floodplain with high intra-annual water level fluctuations. Our main purpose is to quantify the relative importance of deterministic and stochastic processes on the temporal dynamics of fish community, and to investigate if these processes differ between the modified and natural habitats. In natural habitat, inter- and intra- annual variabilities in exogenous physico-chemical factors rising from natural climatic and environmental events, such as fluctuations of water level, $\mathrm{pH}$, salinity and nutrients, are relatively high in comparison with the modified habitat (Bunn \& Arthington 2002). Life histories of native biota, especially the exploitative taxa, are finely tuned to capitalize on the specialized temporal niches associated with this variability (Chase et al. 2011). Consequently, temporal diversity can be promoted through an array of mechanisms, such as migration to exploit resources and escape competition (Shaw 2016), synchronous seasonal reproduction, and seasonal fluctuations in abundance (King et al. 2003). Moreover, habitat modification is likely to create dispersal barriers ( $\mathrm{Li}$ et al. 2020), which may reduce chance colonization by opportunistic species. Thus, our working hypothesis is that the ecological stochastic processes will prevail in the natural sites where dispersal is not constrained and environmental variability is high; and the deterministic processes will dominate the human modified sites, where dispersal is limited by isolation and environment is relatively stable.

\section{Material and methods}

\section{Study Site}

Dongting Lake $\left(111^{\circ} 40^{\prime}-113^{\circ} 10^{\prime} \mathrm{E}, 28 \mathrm{deg} 38^{\prime}-29 \operatorname{deg} 45^{\prime} \mathrm{N}\right)$ is China's second largest freshwater lake. Due to land reclamation, the whole lake has been divided into three sub-lakes (East, South, and West Dongting Lake), which are hydrologically connected through main river channels. Our study site, the West Dongting Lake (111deg57' - 112deg17' E, 28deg47' - 29deg07' N) is the most upstream section (Fig. 1). Water from the Yuan River, the Li River and two escaping channels of Yangtze River flows through West Dongting into South Dongting, and then into East Dongting, finally discharges into the Yangtze (Fig. 1). The prevailing climate in the area is continental subtropical monsoon, with four distinct seasons featuring a warm and humid summer and a cold and dry winter. The annual rainfall ranges from 1,200 to 1,700 mm, of which up to $60 \%$ occurs in summer (Guan et al., 2014).

The West Dongting Lake (WDTL) is a seasonally dynamic system, with large inter- and intra-annual variations in both lake area and water level. In a typical year, the water level increases from the end of March and reaches the highest level in July or August. The water level begins to decrease in September and the lowest level occurs from December to March in the next year. During the summer wet season, water level reaches an average level of $34.30 \mathrm{~m}$ (long-term mean, based 1965-2014 records) at Nanzue hydrological station (Fig. 1 ), and the majority of the area is under water. During the winter dry season, the lake water level decreases to $28.03 \mathrm{~m}$ (long-term mean, based 1965-2014 records). As water levels recede, the Lake divides into four broad habitat types: open water, Carex sedge, Phragmites communis marsh andPopulus nigra plantation. While the first two types of habitat are in relatively natural conditions, the other two, especially thePopulus nigra plantation, are subjected to great degree of artificial modifications.

From late 1980s, plantation of Populus nigra, an introduced fast-growing tree for wood pulp, started in WDTL. To ensure young trees survive the summer flooding, elevated ridges were built with sediments excavated from the nearby lakebed, leaving a network of artificial ditches with depth varies from 1.0 to $2.5 \mathrm{~m}$ (Li et al, 2020). The plantation creates a novel habitat, which has reduced lateral hydrological connectivity, flattened hydrograph (i.e. the average peak water level is greatly reduced), and stable water level during the low water period. In addition, the artificial ridges present a significant barrier for fish movement even during high-water seasons. Another notable change is that the excavation destroyed submerged plants and sedges, resulting in a habitat with less structural complexity. 


\section{Fish survey}

During the period of 2017 - 2019, we surveyed benthic fish at 12 locations across the WDTL, of which 6 were from ditches at Populus nigra plantations, 3 were from Carex sedges and 3 were from open waters (Fig. 1). The year 2017 is considered as a flooding year with maximum water level reaching $36.57 \mathrm{~m}$ at Xiaohezuo (Fig. 2), which was nearly $3 \mathrm{~m}$ higher than the long-term mean maximum water level, while 2018 and 2019 is normal with maximum water level of $33.63 \mathrm{~m}$. For each year, we collected monthly samples from April to October, which covers a completed cycle of water level rise and recession (Fig. 2). In April, the water level is normally high enough to inundate most of the Carex sedges and Phragmites communis marshes. Fish move to the newly flooded areas to breed and forage, taking average of the abundant food sources (King et al. 2003). October is near the end of water recession phase, and water level is high enough to maintain the hydrological connection between channels and Carex sedges.

Fish samples were collected using long fyke nets (length: $30 \mathrm{~m}$, width: $20 \mathrm{~cm}$, mesh size: $10 \mathrm{~mm}$ ). The net is designed by local fisherman, and is efficient for benthic fish sampling in areas with complex habitat structure (e.g. macrophyte with different height, irregular topography). For each sampling events, the net was set at 9:00 -10:00 am and the catch was collected at 9:00 - 10:00 the next day. All fish were transferred to laboratory for identification (to species level) and counting.

\section{Environmental variables}

Before setting the net, temperature, $\mathrm{PH}$ and conductivity (SPC) were measured in site using a YSI multiprobe (YSI, Yellow Springs, Ohio, USA), clearance was also measured using a $20 \mathrm{~cm}$ white Secchi disc. We also collected instantaneous water grab samples to measure total phosphorus (TP), total nitrogen (TN), total suspended solids (TSS), and chlorophyll a (chla). Vertically integrated water samples (sub-samples were taken from the surface, $0.5 \mathrm{~m}, 1 \mathrm{~m}$ and $1.5 \mathrm{~m}$ ) were collected and preserved following USEPA methods (Kopp et al. 1983). Water samples were analyzed in laboratory pursuant to APHA protocols (APHA. 2005).

\section{Assessing ecological stochasticity}

We calculated both spatial and temporal ecological stochasticity to explore the mechanisms shaping $\beta$ diversity patterns in WDTL. We used spatial ecological stochasticity (Ning et al. 2019) to compare the deterministic and stochastic processes shaping benthic fish communities at different habitat types. To quantify the spatial ecological stochasticity, we aggerated all sample sites for each survey occasions within a habitat type to form a metacommunity, assuming that fish can move freely between sampling locations. We used temporal stochasticity to assess the relative importance of deterministic and stochastic processes on community dynamics (see below). To calculate temporal stochasticity, for each site, the monthly community matrices in every sampling year were considered as a metacommunity. Following Chase et al. (2009), we use null models to determine the ecological stochasticity in shaping the benthic fish community. Briefly, the community dissimilarity (we used the abundance-weighted Bray-Curtis index) is compared with the null expectations to quantify ecological stochasticity under different situations (Zhou et al. 2014). In this study, the null communities were generated by randomizing the observed community structure 1,000 times based on the SIM2 algorithm in Gotelli (2000), i.e. species occurrence totals are fixed but all sites within a habitat type are equiprobable. The normalized stochasticity ratio (NST), was then developed with $50 \%$ as the threshold between more deterministic $(<50 \%)$ and more stochastic $(>50 \%)$ assembly. NST ranges from 0 to 1 , where the minimum value is 0 when extremely deterministic assembly drives communities completely the same or totally different, and the maximum value is 1 when completely stochastic assembly makes communities the same as null expectation. The detailed mathematic formulas can be found in Ning et al. (2019). 


\section{Quantify the magnitude and variability of temporal fish community dynamics}

We calculate four metrics to describe patterns of the fish community change: total temporal $\beta$-diversity, stability, synchrony, and variation ratio. These metrics were calculated for each year at every sampling site using the abundance-based community matrix.

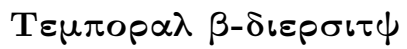

We calculated the total $\beta$-diversity (BDTotal) as the total variance in the community matrix (Legendre \& De Caceres, 2013) using the abundance based Sørensen index with the beta.div function in the "adespatial" package (Dray et al. 2016).

\section{Stability}

Community temporal stability was calculated as $\mu / \sigma$, using the time series of fish catch data collected for each sampling sites, where $\mu$ is the average aggregate abundances of a site across all months and $\sigma$ is the temporal standard deviation in the fish catch of a site (Tilman 1999).

\section{Variance ratio}

Variance ratio (VR) characterizes species covariance patterns in a community (Schluter 1984), which compares the variance of the community $(C)$ as a whole relative to the sum of the individual species abundance $\left(S_{i}\right)$ variances:

$V R=\frac{\sigma^{2}(C)}{\sum_{i=1}^{N} \sigma^{2}\left(S_{i}\right)}(1)$

and

$\sigma^{2}(C)=\sum_{i=1}^{N} \sigma^{2}\left(S_{i}\right)+2 \times \sum_{j<i}^{N} \sigma^{2}\left(S_{i}, S_{j}\right), N$ is the number of species in a community.

If species vary independently (i.e. they do not covary), then the variance ratio will be close to 1 . A variance ratio $<1$ indicates predominately negative species covariance, whereas a variance ratio $>1$ indicates that species generally positively covary (Schluter 1984; Hallett et al. 2014). We tested if the observed variation ratio significantly differs from 1 using null model approach. By randomly selecting different starting points of time series for each species, we generated 10,000 null communities in which species abundances vary independently but within-species auto correlation is maintained (Hallett et al. 2014). The significance is confirmed if the observed variation ratio falls out of the $1^{\text {st }}$ and $3^{\text {rd }}$ percentile of the null distribution.

The calculation of community stability and variation ratio, and significance tests were conducted using the "codyn" package (Hallett et al. 2016) in R (R Core Team 2019).

\section{Synchrony}

Community synchrony provides another measure of species covariance. In study, we defined community synchrony as the degree to which the abundance of fish species in a community rise and fall together through time. Using the community time series of abundance, we calculated community synchrony using the metric of Loreau and de Mazancourt (2008):

$\varphi=\frac{\delta^{2}\left[\sum_{i=1}^{N} S_{i}(t)\right]}{\left(\sum_{i=1}^{N} \delta\left[S_{i}(t)\right]\right)^{2}}(2)$

where $\delta^{2}$ is the variation operator; and $S_{i}(t)$ is the abundance of species $i$ at sampling time $t$. The numerator represents the temporal variance of the aggregate community-level abundance; and denominator is the sum of the population variances squared. $\varphi$ ranges from 0 (perfect asynchrony) to 1 (perfect synchrony) (Loreau and de Mazancourt 2008).

We tested the significance of the observed community synchrony by comparing it with the distribution of synchrony of 10,000 random communities. The random communities were generated using a Monte Carlo 
randomization procedure, which shuffles the columns of the community matrix independently. Community synchrony calculation and test were conducted using the "synchrony" package (Gouhier and Guichard 2014).

\section{Statistical analysis}

To determine if and how community temporal dynamics is affected by environmental factors, we use generalized linear mixture models (GLMM) or generalized additive mixed models (GAMM), to count the possible non-linear relationship (Wood 2006). Starting from the simplest null model with no explanatory variable, we increased the model complexity by adding sequentially independent variables (i.e. TP, TN, TN:TP, Depth, habitat type and sampling year) as either simple or smooth terms. Because the relatively small sample size, we restricted the number of independent variables to three in any single model. For each community dynamic metric, a total of 126 models were fitted. We select and report the best model using leave-one-out cross-validation (loo), which is a fully Bayesian model selection procedure, similar to the widely applicable weighted Akaike's information criterion (Vehtari et al., 2017). Moreover, we also investigated the relationships between stability and diversity, VR and synchrony in the same way to explore the effects of biological interactions on community stability (Hallett et al. 2014). To explore the effects of species diversity on community stability, we aggregated all monthly fish catches for each site and calculated the inverse Simpson's diversity index as community diversity.

The posterior distributions of model parameters were estimated using Markov chain Monte Carlo (MCMC) methods with NUTS sampler implemented in the R package "brms" (version 2.1.0, Bürkner, 2017) by constructing four chains of 100,000 steps, including 50,000-step warm-up periods, so a total of 200,000 post-warm up draws (i.e. $(100,000-50,000) \times 4=200,000)$ are retained to estimate posterior distributions. All modeling procedures are conducted in a Bayesian framework using the programme Stan (Carpenter et al. 2017) implemented in the R package "brms" (Bürkner 2017).

We used the same Bayesian approach to explore the temporal and spatial patterns of NST, and to link NST with environmental factors.

\section{Results}

\section{Overall fish diversity}

We recorded a total of 29 benthic species during 252 surveys over the three-year study. Raw sample species richness ranged from 0 to 13 and average richness at sites was 4.2. There were two occasions that we recorded no fish and these events were discarded in the analysis. The inter- and intra- annual differences in richness were not significant $(\mathrm{p}=0.411$ and 0.542 for monthly and annually richness, t-test), however, the richness difference between habitat types was significant with more species recorded at natural sites (Carex sedges and open waters) than at plantation sites (t-test, $\mathrm{p}=0.002$ and $<0.001$ for Carex sedges and open waters, respectively). For abundance, although there was no significant inter-annual difference $(\mathrm{p}=0.452$, t-test), the catch was significantly higher in May than other months $(\mathrm{p}=0.028$, t-test $)$, coincident with the breeding season. In contrast to richness, fish abundance at Carex sedge sites was significantly higher than that at plantation sites $(\mathrm{P}<0.001$, t-test $)$ and open water sites $(\mathrm{P}=0.044$, t-test $)$, which had comparable abundance $(\mathrm{p}=0.200$, t-test $)$.

\section{Temporal community variation and its drivers}

Synchrony, variance ratio and stability varied greatly across habitat types and between sampling years (Table 1 ). The total $\beta$-diversity showed great spatial and temporal variations (Table 1). Specifically, the temporal variation of benthic fish communities was significantly lower in the flooding year (i.e. 2017) than in normal years (i.e. 2018 and 2019) $(p<0.01$, t-test). In plantations, the total temporal $\beta$-diversity was significantly 
lower than open waters and Carex sedges $(p<0.01$, t-test). Although the difference in temporal $\beta$-diversity was significant ( $p=0.04$, t-test) in the flooding year, it became non-significant in normal years. The best model fitted for temporal $\beta$-diversity included water total phosphorus concentration (TP) and habitat type. This model explained $68 \%$ of the variations of $\beta$-diversity across the sampling sites (Table 2 ). The estimated model coefficient indicated that temporal $\beta$-diversity decreased significantly among the TP gradients, and was significantly lower in plantations than in the natural habitat of Carex sedges and open waters.

The observed synchrony of fish abundance ranged from 0.09 to 0.90 , and in 10 occasions out of the total of 36 , the synchrony was significantly greater than null expectations. These results suggested that most taxa abundances were changing in the same direction, especially in the modified habitat (Table 1).

We did not detect any spatial or temporal patterns in synchrony variations (Table 2). The best model describing the synchrony variation included a single environmental variable (i.e. TN, Table 2), which explained $38 \%$ of the variation in the spatial and temporal variation of community synchrony (Table 2). Total nitrogen had a significantly (at 0.05 level) negative effects on synchrony (the lower $2.5 \%$ and upper $97.5 \%$ intervals do not include zero). Other tested environmental variables such as water depth, TP, and water clearance had no statistically significant relationship to species synchrony.

The calculated VR ranged from $0.30-2.83$ with the majority cases greater than one (Table 1), of which seven were significantly higher than the null VR. Similar to species synchrony, these results indicated that the benthic fish community largely positively covaried, especially at the plantation sites. As for variation ratio, there was no clear spatial or temporal patterns. In addition, no model with environmental variables was better than the null based on the loo model selection procedure.

Community stability had a weakly negative relationship with TN. The relationship was not significantly at 0.05 level (Table 2), but was at 0.1 level (the lower 5\% and upper 95\% interval of the coefficient for TN was -0.12 to -0.01 , data not shown). Like synchrony and VR, other tested environmental variables had no significant impacts on community stability.

\section{Biotic mechanisms of community temporal dynamics}

Species synchrony and the variance ratio were significant predictors for community stability ( $53 \%$ and $66 \%$ of the total variation was explained by the VR and synchrony model, respectively, Table 3 ). In comparison, both $\alpha$ and $\beta$ diversity models had considerably lower explanatory power with a mean Bayes- $R^{2}$ of $0.33(0.30,0.54)$ and $0.23(0.06,0.40)$ (Table 3$)$. This lower performance was also reflected in the relatively larger confidence interval of the response curve, especially when abundance stability was high (Fig. 3). While the modelled relationship with VR and $\beta$ diversity was linear, stability had non-linear relationships with synchrony and $\alpha$ diversity (Table 3 and Fig. 3). Community stability decreased with both VR and synchrony, however, the marginal effect curve of the stability - $\alpha$ diversity was hump-shaped unimodal, suggesting lower stability at both the extreme ends. In contrast, the relationship with $\beta$-diversity was linear and community abundance stability increased significantly with $\beta$-diversity (Table 3, Fig. 3).

Habitat type was also included in these models. The estimated model coefficients suggested that the plantation sites had significantly higher community stability than the sedge sites (the $95 \%$ interval did not include zero for all models, Table 3). However, the difference between plantation and open water was not significant when the effects of species variance rate, synchrony or $\alpha$-diversity were accounted for (zero was within the bracket of the $95 \%$ confidence interval, Table 3 ).

\section{Ecological Stochasticity and its effects on community dynamics}

There were no inter- and intra- annual patterns in spatial ecological stochasticity in benthic fish community. However, there were strong spatial patterns: the modified sites had significantly lower stochasticity than the more natural sites (Fig. 4). The modelled coefficient indicated that ecological stochasticity was the predominant process at natural sites (mean ecological stochasticity greater than 0.50, Fig. 4), whereas 
deterministic processes were prevailing at the modified sites (mean ecological stochasticity was 0.37, Fig. 4). In addition, none of the tested environmental variables had a significant effect on ecological stochasticity and the best regression model included only habitat types as explanation variable. The model had a relatively high Bayse- $R^{2}$ of 0.42 (95\% confidence interval was $\left.0.20-0.57\right)$.

The temporal ecological stochasticity was a strong predictor of all investigated metrics of community temporal dynamics (the mean Bayes- $R^{2}$ and its $95 \%$ confidence interval were $0.41[0.22,0.55], 0.30$ [0.08, 0.50], $0.59[0.42,0.69]$ and $0.67[0.55,076]$ for stability, variance rate, synchrony, and total temporal $\beta$-diversity respectively, Table 4). While the normalized ecological stochasticity had significantly positive effects on species variance rate and synchrony, it had significantly negative effects on community stability (Fig. 5). The effects of stochasticity on temporal $\beta$-diversity was highly non-linear: the $\beta$-diversity increased with stochasticity at low level but stayed more or less stationary when the NST reached $~ 0.4$.

The community stability was significantly higher in the plantation habitat than in the sedge habitat while the difference between plantation and open water was not significant (Table 4). Moreover, community stability was significantly lower in the flooding year of 2017 than in the normal water years of 2018 and 2019. For species synchrony and variance rate, the difference between habitat types was not significant (Table 4). Regarding the yearly variation, species synchrony was significantly lower in flooding year of 2017, and the between-year difference for species variance rate was not significant (Table 4).

\section{Discussion}

Despite decades of study, quantifying the relative importance of deterministic versus stochastic processes in natural community assembly remains a key challenge to ecologists (Dini-Andreote et al. 2015; Tucker et al. 2016), especially in dynamic species-rich landscapes (Ruhí et al. 2017). In this study, we investigated the temporal dynamics of the benthic fish communities in two habitat types (i.e. natural vs modified) within a large river-floodplain system over a water level rising-and-drawdown cycle for three consecutive years. We found distinct assembly processes operating between habitat types, contributing to the variation in observed community dynamics, such as temporal $\beta$-diversity community stability, and species synchrony. Moreover, our results suggested that the nutrient gradients strongly mediate the community assembly processes (Donohue et al. 2009), exhibiting significant effects on temporal community dynamics.

\section{Distinct normalized ecological stochasticity in natural and modified habitats}

Null models have become a valuable tool to infer assembly processes from spatial variations in community structure (Chase et al. 2011; Mori et al. 2015). However, few studies have applied null models to explore the temporal dimension of community turnover. Here, we used the NST based on a null modelling approach (Ning et al. 2019) to quantify the ecological stochasticity using monthly samples of benthic fish community. NST reflects the contribution of stochastic assembly relative to deterministic assembly, providing a better quantitative measure of stochasticity than other randomization-based measurements such as standardized effect size (Ning et al. 2019). While the mean NST was much less than 0.50 in the modified sites, it was greater than 0.50 at the natural sites. These results suggested that deterministic processes, either environmental filtering or biotic interactions or both (Dini-Andreote et al. 2015; Ning et al. 2019) were the main mechanisms underlying the temporal variation in community structure in the modified habitat. In contrast, in the natural sites especially in open waters, stochastic processes, i.e. demographic (e.g. probabilistic dispersal and random birth - death events) and environmental stochasticity (e.g. changes in water level, pH and water clearance) (Chase \& Myers 2011;Shoemaker et al. 2020) could be the prevailing processes driving the temporal structural changes in fish communities.

The lower stochasticity (thus, higher deterministic forcing) in modified habitats could be attributed to many interconnected abiotic and biotic factors. First, modification reduces hydrological connectivity (Li et al. 2020), which depresses temporal variation and creates relatively stable environmental conditions. In turn, 
the stable conditions might trigger environmental filtering and biotic interaction, such as competition. Second, habitat modification cleared submerged macrophytes through excavation of sediment (field observation by authors), leading to further environmental homogenization in terms of structural complexity. Third, modification could impose dispersal limitation through habitat isolation. Dispersal (e.g., random chance for colonization) can be considered as a more stochastic process that induces deviations from expectations based solely on niche theory (Chase and Myers 2011). Studies have shown that ecological connectivity and dispersal play a central role in structuring communities (Cadotte et al. 2006; Vellend et al. 2014). Our findings demonstrated the importance of considering the ensemble of local communities as an integrated metacommunity, and approach which improves our understanding of the processes maintaining biodiversity in complex dynamic landscapes.

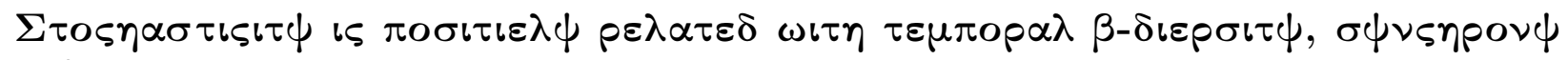

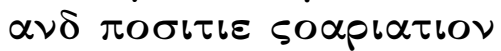

Previous studies have demonstrated that human disruption, such as habitat modification, could cause biotic homogenization (Iacarella et al. 2018), leading to reduced spatial $\beta$-diversity in fish communities in floodplain environments (Quintero et al. 2010; Li et al. 2020). In this study, we found significantly lower temporal $\beta$-diversity of benthic fish communities in the modified plantations than in the natural Carexsedges and open waters. Moreover, a significantly positive relationship between ecological stochasticity and total temporal $\beta$-diversity was confirmed.

Although the difference in species variance rate between habitat types was not significant, species synchrony in communities was generally higher in natural habitats than in the modified sites. In addition, both species synchrony and variance rate were significantly positively related to NST. The higher synchrony in natural habitats can be partially explained by life history theory (Mims \& Olden 2012). Native fish have developed life history strategies adapted to the natural inter- and intra- variations in hydrology (McManamay \& Frimpong 2015). Therefore, the life cycles of native fish, such as reproduction, larval and juvenile survival, migration and movement of young and mature fish, are synchronized with long-term hydrological regime (King et al. 2003). In the plantation, to ensure the survival of young trees, the natural hydrological regime was greatly modified, especially during low water periods ( $\mathrm{Li}$ et al. 2020), resulting an interruption to how native biota respond to environmental cues. There is general agreement that dispersal can synchronise spatially distinct subpopulations (Goldwyn \& Hastings 2008; Vogwill et al. 2009). Therefore, the lower species synchrony in modified habitats could also be attributed to the dispersal limitation.

The community stability, measured by the time series of a univariate index (the sum of fish abundance), was significantly higher in the modified habitat than in natural habitats. It had significantly negative relationships with NST, species synchrony and variance rate. As we have shown that ecological stochasticity could lead to high synchrony and positive covarying (i.e. abundance changes in the same direction) among fish species in river-floodplain system, these negative relationships suggested that temporal asynchrony (i.e. compensatory dynamics, as in Gonzalez and Loreau 2009) helps to stabilize abundance fluctuations in the modified habitats. Thus, the persistence of the generalists such as Carassius auratus was higher at the modified plantation sites, where environmental conditions are relative stable. As the use of a single univariate index can potential give misleading impression of the overall picture of community stability (Death \& Winterbourn 1994), time lag analysis using community distance (Collins et al. 2000 \& 2008; Hallett et al. 2016) can provide insights into community stability (Jones et al. 2017). However, the duration of our dataset is not long enough to have a meaningful analysis using this approach.

\section{Nutrient gradients mediate community temporal dynamics}

Nutrient enrichment affects temporal dynamics of biotic communities (Cook et al. 2018; Avolio et al. 2014), and many studies documented that elevated nutrient levels cause biotic homogenization, reducing the temporal $\beta$-diversity of biotic communities, such as plants (Zhou et al. 2020) and macroinvertebrates 
(Huttunen et al. 2020). We found significant relationships between nutrient level (TN or TP) and community temporal dynamics. Species synchrony and variance rate had negative relationship with TN whereas the temporal $\beta$-diversity decreased significantly with TP. Note that TP and TN were highly correlated (Pearson's $\mathrm{r}=0.73$ ), and we used the variable with the closer relationship in all models. Johnson and Angeler (2014) also found the negative relationship between TP and temporal $\beta$-diversity in a meta-analyses of European fish community diversity. The low phylogenetic diversity and loss of functional diversity in high nutrient conditions might be mediated by periodically hypoxic conditions in floodplain settings (King et al. 2012) or the responses of food sources such as benthic diatoms (Allen 2004; Johnson and Angeler 2014).

\section{Conclusion}

There is a paradigm shift from pattern-based hotspot management to process-based functional approach in biodiversity conservation strategy. The sustenance of goods and services provided by ecosystems requires not only the investigation of biodiversity patterns but also the identification of processes that produce and preserve regional and local biodiversity. Although community ecologists generally agree that ecological metacommunity is structured simultaneously by stochastic and deterministic rules, one assembly rule may be predominant in a given landscape. In this study, we showed that ecological stochasticity prevails in natural habitat whereas deterministic processes dominate in modified environment in a highly dynamic river-floodplain system. In addition, the patterns of community temporal dynamics are consistent with ecological stochasticity. The shift in assembly rules in modified sites are the likely outcomes of the relatively stable environment created by human interruption to hydrological connectivity. First, modification alters hydrological regime, which removes the drawdown phase variability and maintains low water levels to aid production, thereby removing the stochastic effects of periodic habitat exclusion. Second, the flattened hydrograph in the high-water phase creates dispersal barriers, reducing demographic stochasticity such as chance colonization. Third, modification reduces the topographic heterogeneity, which minimises benthic structural variability characteristic of the natural sedge habitats, leading to stronger biotic interactions.

\section{Acknowledgements}

We would like to thank the staffs at the West Dongting Lake National Nature Reserve and Xiao Yayu, Zhao Changbin, and Guo Min for their help in data collection. This study was supported by the National Key Research and Development Program of China (Grant Number 2017YFC0405303), the Fundamental Research Funds for the Central Universities (Grant Numbers 2017ZY15).

\section{Reference}

1. American Public Health Association, A.P.H.A. (1998). Standard Methods for the Examination of Water and Wastewater. American Public Health Association Inc., Washington, DC.

2. Allan, J.D., (2004). Landscapes and riverscapes: the influence of land use on stream ecosystems. Annu. Rev. Ecol. Syst. 35, 257-284.

3. Anderson, M.J. et al. (2011). Navigating the multiple meanings of $\beta$ diversity: a roadmap for the practicing ecologist. Ecology letters, 14, 19-28.

4. Avolio, M.L. et al. (2014). Changes in plant community composition, not diversity, during a decade of nitrogen and phosphorus additions drive above-ground productivity in a tallgrass prairie. Journal of Ecology , 102, 1649-1660.

5. Bayley, P. (1995). Understanding Large River-Floodplain Ecosystems: Significant economic advantages and increased biodiversity and stability would result from restoration of impaired systems.BioScience , 45, 153-158. 
6. Baselga A. (2010). Partitioning the turnover and nestedness components of beta diversity. Global ecology and biogeography, 19, 134-143.

7. Bunn, S.E. and Arthington, A.H. (2002). Basic principles and ecological consequences of altered flow regimes for aquatic biodiversity. Environmental management, 30, 492-507.

8. Bürkner P. C. (2017). Advanced Bayesian multilevel modeling with the R package brms. arXiv preprint arXiv , 1705.11123.

9. Castello L, Macedo M. N. (2016). Large-scale degradation of Amazonian freshwater ecosystems. Global Change Biology , 22, 990-1007.

10. Carpenter B. et al. (2017). Stan: A probabilistic programming language. Journal of statistical software ,76(1).

11. Cadotte, M.W. et al. (2006). The effects of resource enrichment, dispersal, and predation on local and metacommunity structure. Oecologia , 149, 150-157.

12. Chase, J.M. et al. (2011). Using null models to disentangle variation in community dissimilarity from variation in $\alpha$-diversity.Ecosphere, 2, 1-11.

13. Chase, J.M. and Myers, J.A. (2011). Disentangling the importance of ecological niches from stochastic processes across scales.Philosophical transactions of the Royal Society B: Biological sciences , 366, 2351-2363.

14. Chase, J.M. et al. (2009). Predators temper the relative importance of stochastic processes in the assembly of prey metacommunities.Ecology letters , 12, 1210-1218.

15. Collins, S. L. et al. (2000). A method to determine rates and patterns of variability in ecological communities. Oikos, 91, 285-293.

16. Collins, S.L. et al. (2008). Rank clocks and plant community dynamics.Ecology, 89, 3534-3541.

17. Cook, S.C. et al. (2018). Freshwater eutrophication drives sharp reductions in temporal beta diversity. Ecology , 99, 47-56.

18. Cottenie, K. (2005). Integrating environmental and spatial processes in ecological community dynamics. Ecology letters , 8, 1175-1182.

19. Daniel, J. et al. (2019). Stochastic and deterministic processes drive wetland community assembly across a gradient of environmental filtering. Oikos , 128, 1158-1169.

20. Death, R.G. and Winterbourn, M.J., (1994). Environmental stability and community persistence: a multivariate perspective. Journal of the North American Benthological Society, 13, 125-139.

21. Dini-Andreote, F. et al. (2015). Disentangling mechanisms that mediate the balance between stochastic and deterministic processes in microbial succession. Proceedings of the National Academy of Sciences $, 112,1326-1332$.

22. Dirzo, R. et al. (2014). Defaunation in the Anthropocene.Science, 345, 401-406.

23. Donohue, I. et al. (2009). Nutrient enrichment homogenizes lake benthic assemblages at local and regional scales. Ecology, 90, 3470-3477.

24. Dray, S. et al. (2016). adespatial: Multivariate multiscale spatial analysis. $R$ package version $0.0,3$.

25. Economo, E.P. (2011). Biodiversity conservation in metacommunity networks: linking pattern and persistence. The American Naturalist, 177, 167-180.

26. Fontenot Q. C. et al . (2001). Effects of Environmental Hypoxia Associated with the Annual Flood Pulse on the Distribution of Larval Sunfish and Shad in the Atchafalaya River Basin, Louisian. Transactions of the American Fisheries Society, 130, 107-116.

27. Guan L. et al . (2016). Optimizing the timing of water level recession for conservation of wintering geese in Dongting Lake, China. Ecological Engineering , 88, 90-98.

28. Garcia-Moreno, J. et al. (2014). Sustaining freshwater biodiversity in the Anthropocene. In: The global water system in the Anthropocene, [ed(s).] [Bhaduri A., Bogardi J., Leentvaar J., Marx S.]. Springer, Cham, 247-270.

29. Gaston, K.J. and Chown, S.L. (2005). Neutrality and the niche.Functional Ecology, 19, 1-6.

30. Gelman, A. et al. (2019). R-squared for Bayesian regression models. The American Statistician , 73, 307-309.

31. Goldwyn, E.E. and Hastings, A., (2008). When can dispersal synchronize populations?. Theoretical 
population biology, 73(3), pp.395-402.

32. Gonzalez, A. and Loreau, M., 2009. The causes and consequences of compensatory dynamics in ecological communities. Annu. Rev. Ecol. Evol. Syst. , 40, 393-414.

33. Gonzalez, A. et al. (2016). Estimating local biodiversity change: a critique of papers claiming no net loss of local diversity. Ecology , 97, 1949-1960.

34. Gotelli, N.J. et al. (2000). Null model analysis of species co-occurrence patterns. Ecology , 81, 2606-2621.

35. Gouhier, T.C. and Guichard, F. (2014). Synchrony: quantifying variability in space and time. Methods in Ecology and Evolution, 5, 524-533.

36. Gravel, D. et al. (2006). Reconciling niche and neutrality: the continuum hypothesis. Ecology letters $, 9,399-409$.

37. Hallett, L.M. et al. (2014). Biotic mechanisms of community stability shift along a precipitation gradient. Ecology , 95, 1693-1700.

38. Hallett, L.M. et al. (2016). codyn: an R package of community dynamics metrics. Methods in Ecology and Evolution , 7, 1146-1151.

39. Heino J et al. (2015). Metacommunity organisation, spatial extent and dispersal in aquatic systems: patterns, processes, and prospects.Freshwater Biology , 60, 845-869.

40. Hubbell SP. (2001). The Unified Neutral Theory of Biodiversity and Biogeography. Princeton University Press, Princeton.

41. Huttunen, K.L. (2020). Excess of nitrogen reduces temporal variability of stream diatom assemblages. Science of The Total Environment, 713, 136630.

42. Iacarella J. C. et al.(2018). Anthropogenic disturbance homogenizes seagrass fish communities. Global change biology, 24, 1904-1918.

43. Jones, S.K. et al. (2017). Species reordering, not changes in richness, drives long-term dynamics in grassland communities.Ecology letters , 20, 1556-1565.

44. Johnson, R. K., and Angeler, D. G. (2014). Effects of agricultural land use on stream assemblages: Taxon-specific responses of alpha and beta diversity. Ecological Indicators , 45, 386-393.

45. Jia Y. et al. (2020). Processes shaping wintering waterbird communities in an intensive modified landscape: Neutral assembly with dispersal limitation and localized competition. Ecological Indicators. $114,106330$.

46. Kupferberg S. J. et al. (2012). Effects of flow regimes altered by dams on survival, population declines, and range-wide losses of California river-breeding frogs. Conservation Biology , 26, 513-524.

47. Kéfi, S. et al. (2019). Advancing our understanding of ecological stability. Ecology letters , 22, 13491356.

48. King, A.J. et al. (2003). Fish recruitment on floodplains: the roles of patterns of flooding and life history characteristics. Canadian Journal of Fisheries and Aquatic Sciences , 60, 773-786.

49. King, A. J. et al. (2012). Short-term effects of a prolonged blackwater event on aquatic fauna in the Murray River, Australia: considerations for future events. Marine and Freshwater Research , 63, 576-586.

50. Korhonen, J.J. et al. (2010). A quantitative analysis of temporal turnover in aquatic species assemblages across ecosystems.Ecology, 91, 508-517.

51. Kopp J. F. et al. (1983). Methods for chemical analysis of water and wastewater. United States Environmental Protection Agency: Washington, DC.

52. Lu C. et al . (2018). Shifts in river-floodplain relationship reveal the impacts of river regulation: A case study of Dongting Lake in China. Journal of Hydrology, 2018, 559, 932-941.

53. Li, B. et al. (2020). Anthropogenic habitat alternation significantly decreases $\alpha$ - and $\beta$-diversity of benthopelagic metacommunity in a large floodplain lake. Hydrobiologia 847, 293-307.

54. Leibold, M.A. et al. (2004). The metacommunity concept: a framework for multi-scale community ecology. Ecology letters, 7, 601-613.

55. Legendre P. and De Cáceres M. Beta diversity as the variance of community data: dissimilarity coefficients and partitioning.Ecology letters , 2013, 16, 951-963. 
56. Legendre, P., 2019. A temporal beta-diversity index to identify sites that have changed in exceptional ways in space-time surveys.Ecology and Evolution , 9, 3500-3514.

57. Levi, T. et al. (2019). Tropical forests can maintain hyperdiversity because of enemies. Proceedings of the National Academy of Sciences, 116, 581-586.

58. Loreau, M. and de Mazancourt, C. (2008). Species synchrony and its drivers: neutral and nonneutral community dynamics in fluctuating environments. The American Naturalist , 172, 48-66.

59. Magurran, A.E. et al. (2019). Temporal $\beta$ diversity-A macroecological perspective. Global Ecology and Biogeography , 28, 1949-1960.

60. Magurran, A.E. (2018). Divergent biodiversity change within ecosystems. Proceedings of the National Academy of Sciences , 115, 1843-1847.

61. McGill, B.J. (2003). A test of the unified neutral theory of biodiversity. Nature , 422, 881-885.

62. McGill, B.J. et al. (2015). Fifteen forms of biodiversity trend in the Anthropocene. Trends in ecology E evolution, 30, 104-113.

63. McManamay, R.A. and Frimpong, E.A., (2015). Hydrologic filtering of fish life history strategies across the United States: implications for stream flow alteration. Ecological Applications , 25, 243-263.

64. Mims, M.C. and Olden, J.D., (2012). Life history theory predicts fish assemblage response to hydrologic regimes. Ecology, 93, 35-45.

65. Mori, A.S. et al. (2013). Community assembly processes shape an altitudinal gradient of forest biodiversity. Global Ecology and Biogeography, 22, 878-888.

66. Mori, A.S. et al. (2015). Null model approaches to evaluating the relative role of different assembly processes in shaping ecological communities. Oecologia , 178, 261-273.

67. Mori, A.S. et al. (2018). $\beta$-diversity, community assembly, and ecosystem functioning. Trends in Ecology \& Evolution, 33, 549-564.

68. Musters, C.J.M. et al.(2019), Spatial and temporal homogenisation of freshwater macrofaunal communities in ditches. Freshwater Biology, 64, 2260-2268.

69. Ning, D. et al. (2019). A general framework for quantitatively assessing ecological stochasticity. Proceedings of the National Academy of Sciences , 116, 16892-16898.

70. Olden J. D. and Naiman R. J. (2010). Incorporating thermal regimes into environmental flows assessments: modifying dam operations to restore freshwater ecosystem integrity. Freshwater Biology , 2010, $55,86-107$.

71. Palmer, M. and Ruhí, A. (2019). Linkages between flow regime, biota, and ecosystem processes: Implications for river restoration.Science, 365, 2087.

72. Poff L. R. et al . (1997). The Natural Flow Regime: A Paradigm for River Conservation and Restoration. BioSicence, 47, 769-784.

73. Poff L. R. et al . (2006). Hydrologic variation with land use across the contiguous United States: Geomorphic and ecological consequences for stream ecosystems. Geomorphology, 79, 264-285.

74. Pander, J. et al. (2015). Succession of fish diversity after reconnecting a large floodplain to the upper Danube River.Ecological engineering , 75, 41-50.

75. Quintero, C. et al. (2010). Effects of anthropogenic habitat disturbance on local pollinator diversity and species turnover across a precipitation gradient. Biodiversity and Conservation, 19, 257-274.

76. Reid, A.J., et al . (2019). Emerging threats and persistent conservation challenges for freshwater biodiversity.Biological Reviews , 94, 849-873.

77. Rosset, V. et al. (2017). Do lentic and lotic communities respond similarly to drying?. Ecosphere , 8(7), p.e01809.

78. Ruhí, A. et al. (2017). Interpreting beta-diversity components over time to conserve metacommunities in highly dynamic ecosystems. Conservation Biology , 31, 1459-1468.

79. Rosindell, J. et al. (2012). The case for ecological neutral theory. Trends in ecology \& evolution , 27, 203-208.

80. Stewart G, and Harper B. (2002). Barmah-Millewa forest environmental water allocation. Water Science and Technology , 45, 217-223.

81. Shaw, A.K., 2016. Drivers of animal migration and implications in changing environments. Evolutionary 
Ecology, 30, 991-1007.

82. Shoemaker, L.G. et al. (2020). Integrating the underlying structure of stochasticity into community ecology. Ecology , 101, 02922.

83. Silvertown, J. (2004). Plant coexistence and the niche. Trends in Ecology \& evolution, 19, 605-611.

84. Schluter, D. (1984). A variance test for detecting species associations, with some example applications. Ecology , 65, 998-1005.

85. Tilman, D. 1999. The Ecological Consequences of Changes in Biodiversity: A Search for General Principles. Ecology. 80, 1455-1474.

86. Tilman, D., et al. (2006). Biodiversity and ecosystem stability in a decade-long grassland experiment. Nature, 441, 629-632.

87. Tucker, C.M. et al. (2016). Differentiating between niche and neutral assembly in metacommunities using null models of $\beta$-diversity. Oikos , 125, 778-789.

88. Vörösmarty, C. et al . (2010). Erratum: Global threats to human water security and river biodiversity.Nature, $467,555-561$.

89. Vellend, M. et al. (2014). Assessing the relative importance of neutral stochasticity in ecological communities. Oikos, 123, 1420-1430.

90. Vellend, M. et al. (2017). Estimates of local biodiversity change over time stand up to scrutiny. Ecology , 98, 583-590.

91. Vehtari A, et al. (2017). Practical Bayesian model evaluation using leave-one-out cross-validation and WAIC. Statistics and computing, 2017, 27, 1413-1432.

92. Vogwill T. et al. (2009). Dispersal and natural enemies interact to drive spatial synchrony and decrease stability in patchy populations. Ecology Letters , 12, 1194-1200.

93. Wood S. N. (2006). Low-rank scale-invariant tensor product smooths for generalized additive mixed models. Biometrics , 62, 1025-1036.

94. Xia, S. et al . (2017). Restriction of Herbivorous Waterbird Distributions in the Middle and Lower Yangtze River Floodplain in View of Hydrological Isolation. Wetlands, 37, 79-88.

95. Xu, X. et al. (2018). Ecosystem services trade-offs and determinants in China's Yangtze River Economic Belt from 2000 to 2015.Science of the Total Environment ,634, 1601-1614.

96. Yurkonis, K.A. et al. (2005). Invasion impacts diversity through altered community dynamics. Journal of ecology, 93, 1053-1061.

97. Zhou, J. et al. (2014). Stochasticity, succession, and environmental perturbations in a fluidic ecosystem. Proceedings of the National Academy of Sciences , 111, 836-845.

98. Zhou, M. et al. (2020). Plant community temporal stability in response to nitrogen addition among different degraded grasslands.Science of The Total Environment , 729, 138886.

\section{Hosted file}

Tables.docx available at https://authorea.com/users/343066/articles/469757-deterministicand-stochastic-processes-in-natural-and-modified-floodplain-habitats-consequences-forfish-community-temporal-dynamics

\section{Hosted file}

Figures.docx available at https://authorea.com/users/343066/articles/469757-deterministicand-stochastic-processes-in-natural-and-modified-floodplain-habitats-consequences-forfish-community-temporal-dynamics 